\title{
Identification of prognostic inflammatory factors in colorectal liver metastases
}

\author{
Trevor D Hamilton', Derek Leugner ${ }^{2}$, Karen Kopciuk², Elijah Dixon ${ }^{1}$, Francis R Sutherland ${ }^{1}$ and Oliver F Bathe ${ }^{1,3^{*}}$
}

\begin{abstract}
Background: The modified Glasgow Prognostic Score (mGPS) has been reported to be an important prognostic indicator in a number of tumor types, including colorectal cancer (CRC). The features of the inflammatory state thought to accompany elevated C-reactive protein (CRP), a key feature of MGPS, were characterized in patients with colorectal liver metastases. Additional inflammatory mediators that contribute to prognosis were explored.

Methods: In sera from 69 patients with colorectal liver metastases, a panel of 42 inflammatory mediators were quantified as a function of CRP levels, and as a function of disease-free survival. Multivariate statistical methods were used to determine association of each mediator with elevated CRP and truncated disease-free survival.

Results: Elevated CRP was confirmed to be a strong predictor of survival (HR 4.00, $p=0.001$ ) and recurrence (HR 3.30, $p=0.002$ ). The inflammatory state associated with elevated CRP was comprised of raised IL-1 $\beta, I L-6, I L-12$ and IL-15. In addition, elevated IL-8 and PDGF-AB/BB and decreased eotaxin and IP-10 were associated with worse disease-free and overall survival.

Conclusions: Elevated CRP is associated with a proinflammatory state. The inflammatory state is an important prognostic indicator in CRC liver metastases. The individual contributions of tumor biology and the host to this inflammatory response will require further investigation.
\end{abstract}

Keywords: Colorectal, Cancer, Liver, Metastases, Inflammatory, CRP, Cytokine, Prognostic, Surgery

\section{Background}

Colorectal cancer (CRC) is the second leading cause of cancer death in the western world. The liver is the most common site of metastases, affecting approximately 25-35\% [1]. Liver metastases are the most common cause of death in CRC patients [1]. Although there are a number of therapies for treating metastatic CRC isolated to the liver, including ablative therapies and systemic chemotherapy, resection remains the mainstay of treatment for potential cure. With resection, survivals have steadily improved with advances in surgical technique and systemic therapy. In more recent series, median survival is now greater than 50 months and 5-year survival exceeds $50 \%$ [2-4].

With the expansion of therapeutic options, it is becoming increasingly important to select the best treatment

\footnotetext{
* Correspondence: bathe@ucalgary.ca

'Department of Surgery, University of Calgary, Calgary, AB, Canada ${ }^{3}$ Department of Oncology, University of Calgary, 1331-29th St NW, Calgary T2N 4N2, AB, Canada

Full list of author information is available at the end of the article
}

strategy comprising various sequences of systemic therapy and surgery. For example, if a subset of individuals is known to have short disease-free survivals after resection, systemic therapy may play a more important role, either instead of resection or as an adjunct to resection. To this end, clinical risk scores have been developed to stratify patients by prognosis [5-13]. Clinical risk scores are easily employed because the data are readily available from standard preoperative tests employed in clinical practice. However, they shed little insight on the underlying biology of the disease, which is integral to the formulation of new therapeutic strategies.

The modified Glasgow Prognostic Score (mGPS) [14] is a rudimentary means to derive a picture of the overall biological state. The mGPS, based on serum C-reactive protein (CRP) and albumin, was described by McMillan and coworkers [15]. Several series have demonstrated that mGPS effectively stratifies patients by prognosis in CRC [16,17], including CRC liver metastases [18-21]. mGPS also effectively prognosticates in cancers of the

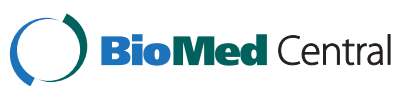


lung, esophagus, pancreas, breast, ovary, bladder and kidney [22-28]. mGPS is thought to reflect the inflammatory state, although the nature of the inflammatory state has not yet been characterized, and the contributions of tumor and host to this inflammatory state have not been delineated.

Understanding the underlying biology of mGPS may leverage its use as a tool to make treatment decisions. That is, with a better comprehension of the inflammatory pathways associated with poor clinical outcomes, it may be possible to redirect the inflammatory response to a more favorable course. The aim of the present study was to characterize the inflammatory state associated with elevated CRP, the main parameter influencing mGPS. A panel of 42 inflammatory mediators, including cytokines and chemokines, were evaluated as a function of CRP. In addition, other important prognostic inflammatory mediators were identified, which may further refine any inflammation-based prognostic scoring system.

\section{Methods}

This study was approved by the Conjoint Health Research Ethics Board at the University of Calgary (Ethics ID E21805). Clinically annotated serum samples were collected prospectively from consented patients who underwent surgery for resection of liver metastases. All patients were treated at the Foothills Medical Centre, a tertiary referral centre, between 2006 and 2011. Patients with extrahepatic disease, or any acute inflammation or sepsis were specifically excluded. Surgical pathology was reviewed for all patients, and confirmed all had colorectal adenocarcinoma. Samples were collected in a plastic gold top Vacutainer tube (BD Biosciences), which contained a clot activator and a gel for serum separation. Samples were processed within 6 hours of collection, then frozen at $-20^{\circ} \mathrm{C}$ until the time of analysis. All samples were collected from patients who had fasted, prior to surgery.

The inflammatory state was characterized in sera using a multiplexed protein bead assay (Luminex, Austin, TX), which measured 42 different inflammatory mediators. Two cytokines (IL-3 and IL-9) were removed from the subsequent analysis due to their high number of zero values (69/70 and 68/70, respectively).

\section{Data analysis}

Patients were allocated to one of two groups, based on a CRP cutpoint level of $10 \mathrm{mg} / \mathrm{L}$, as reported by others [14-16,18,19]. Descriptive statistics were utilized to characterize the clinical parameters of groups: unpaired $t$-tests with unequal variances assumed (Welch's $t$-test) were used to compare means, and Fisher's exact tests were used to compare categorical variables. Quarter-root transformations were employed to improve data symmetry while preserving zero scores assigned to cytokine values below the assay detection limit. All tests of significance were 2 -sided and a $p$-value less than 0.05 was considered a priori to represent statistical significance between groups of patients in these univariate analyses.

To identify individual cytokines associated with elevated CRP, two-sample $t$-tests as well as penalized logistic regression methods were applied based on the elastic net approach $[29,30]$. Multiple testing adjustment for the two-sample $t$-tests was taken into account with the False Discovery Rate via the Significance Analysis Microarrays (SAM) program, which also provides an estimated $p$-value cutoff for a specified false discovery rate [31].

Kaplan-Meier survival plots and Cox Proportional Hazards regression models were used to evaluate the association between cytokines and disease-specific survival. The primary endpoints for that analysis were disease-free survival and overall survival, calculated from date of surgery. Patients without recurrence were censored at their date of last follow-up.

\section{Results}

Inflammatory mediators associated with elevated CRP

Serum inflammatory mediators were evaluated in 70 patients who were considered for surgery. One patient who underwent resection had a recent tooth infection at time of serum sample collection and consequently was excluded from analysis. CRP was measured in the 69 patients with CRC liver metastases and the inflammatory profile was evaluated to determine the nature of the inflammatory response associated with elevated CRP.

The median age for the entire cohort was 61 years (range 29-86), and of those assessed there were 47 males (68\%) and 22 females (32\%). The median CEA was 5.0 (range 0.6-682). Liver metastases were synchronous in 36 patients (52\%). Of those patients with synchronous liver metastases, all had undergone resection of the primary tumor prior to liver resection (and prior to blood collection); no patients were treated with simultaneous colon and liver resections.

A total of 40 inflammatory mediators were tested for their association with elevated CRP (Additional file 1: Table S1). By univariate analysis, there were 4 cytokines that were strongly associated with an elevated CRP: IL-1 $\beta$, IL-6, IL-12, and IL-15 (Table 1). After adjusting for multiple hypothesis testing using the false discovery rate (FDR), 3 cytokines, IL-1 $\beta$, IL-12, and IL-15 were found to be significantly associated with elevated CRP (Table 1).

\section{Prognostic inflammatory profile in resected liver metastases}

To understand which inflammatory mediators were of biological significance, we evaluated mediators associated with recurrence and overall survival. For this analysis, only patients that had a resection were evaluated. Of the 69 patients initially assessed, four patients had progression of disease and did not proceed to surgery, and 13 
Table 1 Cytokines significantly associated with elevated CRP using univariate and multiple hypothesis testing analysis

\begin{tabular}{lcc}
\hline Cytokine & Univariate analysis (p) & SAM (q) \\
\hline IL-1 $\beta$ & 0.023 & 0.000 \\
IL-6 & 0.004 & 0.636 \\
IL-12 & 0.041 & 0.000 \\
IL-15 & 0.006 & 0.000 \\
\hline
\end{tabular}

IL - Interleukin.

SAM - Significance analysis of microarrays.

patients were found to have unresectable disease; these were excluded from further analysis. The baseline clinical and pathologic characteristics of patients in the study who had a liver resection are represented in Table 2 . A total of 27 patients $(52 \%)$ had a normal CRP $(<10 \mathrm{mg} / \mathrm{L})$ and 25

Table 2 Clinical and pathologic characteristics of resected patients with normal and elevated CRP levels

\begin{tabular}{|c|c|c|c|c|c|}
\hline & \multicolumn{2}{|c|}{$C R P<10$} & \multicolumn{2}{|c|}{$\mathrm{CRP} \geq 10$} & \multirow[b]{2}{*}{ P-valu } \\
\hline & $\mathrm{n}$ & $\%$ & $n$ & $\%$ & \\
\hline \multicolumn{6}{|l|}{ Age (years) } \\
\hline$<65$ & 15 & 55.6 & 15 & 60.0 & 0.785 \\
\hline$\geq 65$ & 12 & 44.4 & 10 & 40.0 & \\
\hline \multicolumn{6}{|l|}{ Gender } \\
\hline Male & 18 & 66.7 & 16 & 64.0 & 1.000 \\
\hline Female & 9 & 33.3 & 9 & 36.0 & \\
\hline \multicolumn{6}{|c|}{ Neoadjuvant chemo } \\
\hline Yes & 13 & 48.1 & 15 & 62.5 & 0.400 \\
\hline No & 14 & 51.9 & 9 & 37.5 & \\
\hline \multicolumn{6}{|c|}{ Adjuvant chemo } \\
\hline Yes & 16 & 66.7 & 14 & 66.7 & 1.000 \\
\hline No & 8 & 33.3 & 7 & 33.3 & \\
\hline \multicolumn{6}{|c|}{ CEA (ng/mL) } \\
\hline$<20$ & 15 & 68.2 & 19 & 79.2 & 0.508 \\
\hline$\geq 20$ & 7 & 31.8 & 5 & 20.8 & \\
\hline \multicolumn{6}{|c|}{ Disease-free interval (months) } \\
\hline$<12$ & 21 & 77.8 & 14 & 56.0 & 0.140 \\
\hline$\geq 12$ & 6 & 22.2 & 11 & 44.0 & \\
\hline \multicolumn{6}{|c|}{ Resection margin } \\
\hline Negative & 25 & 92.6 & 18 & 72.0 & 0.071 \\
\hline Positive & 2 & 7.4 & 7 & 28.0 & \\
\hline \multicolumn{6}{|c|}{ Number of tumors } \\
\hline 1 & 14 & 53.8 & 12 & 54.5 & 1.000 \\
\hline$>1$ & 12 & 46.2 & 10 & 45.5 & \\
\hline \multicolumn{6}{|c|}{ Largest tumor size $(\mathrm{cm})$} \\
\hline$<5$ & 22 & 84.6 & 15 & 68.2 & 0.301 \\
\hline$\geq 5$ & 4 & 15.4 & 7 & 31.8 & \\
\hline
\end{tabular}

CRP - C-Reactive Protein.

CEA - Carcinoembryonic Antigen.

Some missing data. patients had an elevated CRP $(\geq 10 \mathrm{mg} / \mathrm{L})$. Liver resection was performed in 34 males (65\%) and 18 females (35\%). The median age was 61 years (range 29-86). Neoadjuvant chemotherapy was administered in 28 patients (54\%), adjuvant chemotherapy was administered in 30 patients (58\%). Median CEA was 3.2 (range 0.6-134). Liver metastases were synchronous in 28 patients (54\%). In 43 patients (83\%), R0 resections were achieved; the remainder had positive microscopic margins. Solitary metastases were resected in 50\%; up to 7 lesions were resected in any individual. The median tumor size was $3.5 \mathrm{~cm}$ (range 0.5-9.0). There was no significant difference in any of these factors between the group with a normal CRP and the group with elevated CRP (Table 2). Specifically, CRP levels did not vary as a function of chemotherapy, CEA level, or number and size of metastases.

Median follow-up for patients still alive was 20 months (range 1-49 months). During follow-up, 15 patients (28\%) died, and 31 patients (58\%) developed recurrent disease. The median overall survival in patients with normal and elevated CRP was 79 months and 41 months, respectively (Figure 1A). However, this difference was not statistically significant by log rank test. The median disease-free survival in patients with normal CRP was 23 months and, in patients with elevated CRP, was 13 months (Figure 1B). Again, this was not statistically significant by log rank test, due to the small sample size. However, when CRP was analyzed as a continuous variable (as opposed to a binary variable), it was found to be a strong predictor of overall survival (hazard ratio 4.00; Table 3) and disease-free survival (hazard ratio 3.30 , Table 4 ).

Finally, we sought to determine if any other inflammatory changes (in addition to those associated with elevated CRP) were prognostic. Elevated levels of IL- 8 and PDGF-AB/BB were associated with a worse overall survival by univariate analysis (Table 3). After adjusting for multiple hypothesis testing, only CRP and PDGR-AB/BB were significant. In contrast, elevated levels of eotaxin and IP-10 were associated with lower rates of recurrence (Table 4). However, after adjusting for multiple hypothesis testing, only CRP was significantly associated with recurrence.

\section{Discussion}

As therapeutic options become more numerous, it becomes increasingly important to develop tools to select the appropriate treatment regimen. In the case of resectable liver metastases, clinical risk scores (employing factors such as CEA, size and number of metastases, disease-free interval, nodal status, margins) effectively stratify most patients by risk of recurrence and survival [5-13]. When encountering a patient with a high-risk score, it may prompt the clinician to defer surgery and/or administer systemic therapy. Similarly, the mGPS has been described to effectively prognosticate for CRC and for CR liver metastases [16-21]. Indeed, 


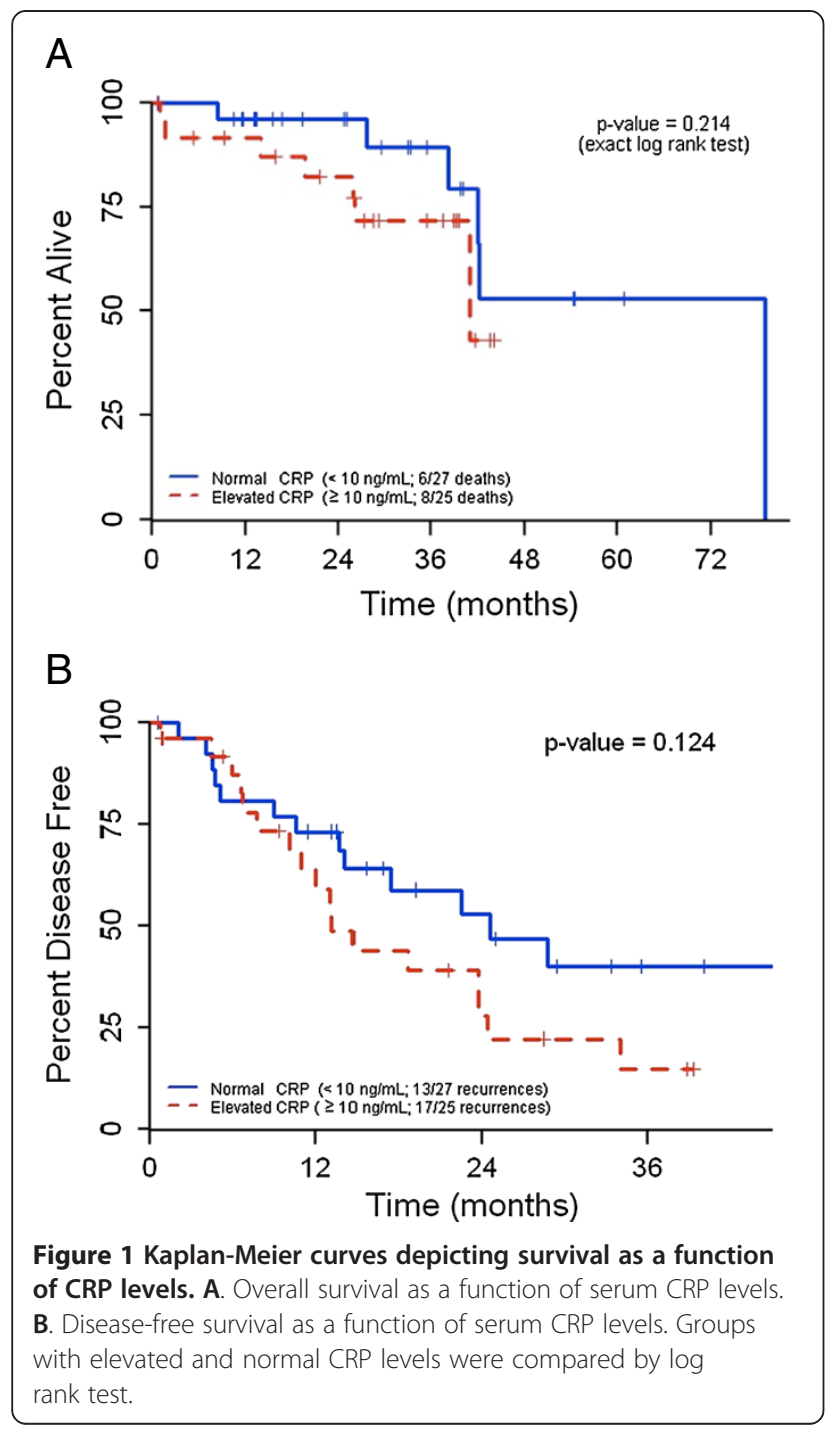

our small series confirms that CRP levels relate to diseasefree and overall survival. mGPS is relatively simple to apply and presumably reflects underlying tumor and/or host biology, although it is not entirely clear how this will affect clinical management.

mGPS is thought to reflect the inflammatory response secondary to malignancy, although the inflammatory response has not been confirmed or characterized. Others have described changes in circulating inflammatory mediators associated with CRC, including elevated IL-2, IL-6,

Table 3 Inflammatory mediators significantly predictive of median overall survival

\begin{tabular}{lccc}
\hline Cytokine & Hazard ratio & $\mathbf{9 5 \% ~ C l}$ & P-value \\
\hline CRP & 4.00 & $1.70-9.31$ & 0.001 \\
IL-8 & 4.96 & $1.35-17.6$ & 0.014 \\
PDGF-AB/BB & 3.16 & $1.42-6.90$ & 0.005 \\
\hline
\end{tabular}

CRP - C-Reactive Protein.

PDGF - Platelet Derived Growth Factor.
Table 4 Inflammatory mediators significantly predictive of median disease-free survival

\begin{tabular}{lccc}
\hline Cytokines & Hazard ratio & $\mathbf{9 5 \% ~ C l}$ & P-value \\
\hline CRP & 3.30 & $1.56-7.00$ & 0.002 \\
Eotaxin & 0.28 & $0.11-0.79$ & 0.014 \\
IP-10 & 0.41 & $0.17-0.96$ & 0.041 \\
\hline
\end{tabular}

CRP - C-Reactive Protein.

IP - Interferon inducible-protein.

and IL-8 without evaluating whether these relate to mGPS [32-34]. More recently, McMillan's group has linked mGPS with IL-6 and tumor necrosis in patients with primary resected colorectal cancer [35]. The current study extends on that work in that a more comprehensive panel of inflammatory mediators was assessed as a function of mGPS. We have demonstrated that elevated CRP is associated with increases in IL- $1 \beta$, IL- 6 , IL- 12 and IL- 15 by univariate analysis. All of these cytokines with the exception of IL-6 remained significantly associated with high CRP levels on multivariate analysis. In general, this confirms that a high mGPS is reflective of a proinflammatory state, with features of a Th1 response.

In addition, a number of other inflammatory mediators (elevated IL-8 and PDGF-AB/BB, as well as depressed eotaxin and IP-10) were found to be independently associated with increased recurrence or truncated survival. IL-8 is a proinflammatory chemokine but is also overexpressed on metastatic colon cancer cells [36], as well as other cancer cells [37]. In the context of cancer, IL-8 is proangiogenic, attracts neutrophils to tumor, and has a number of other functions that encourage tumor growth and metastasis. It may also confer chemoresistance [37]. Colon cancer is associated with elevated platelet concentrations of PDGF [38], presumably reflecting a proangiogenic state. Its expression has not been previously reported to discriminate biologically meaningful subsets. Eotaxin, a chemokine that recruits eosinophils, has been reported to be expressed at higher levels in CRC compared to normal tissue [39,40], although circulating eotaxin is decreased in CRC patients compared to disease-free controls and progressively decreases with more advanced disease [40]. IP-10 is a chemokine that plays a role in the chemoattraction of monocytes, endothelial cells and fibroblasts; it is also an inhibitor of angiogenesis. It is inversely correlated with growth and metastasis in experimental models $[41,42]$. One previous study has shown that low circulating levels of IP-10 correlate with poor prognosis in CRC [43].

In our analysis of cytokines related to prognosis, we did not formally take into account the effect of clinicopathological factors because we could not detect any relationship between CRP levels and because of the limited sample size. One potential confounding factor in our series is the administration of chemotherapy; approximately half of patients had neoadjuvant chemotherapy. Interestingly, 
there was no difference in CRP levels in patients who did and did not receive chemotherapy. In fact, none of the clinical or pathological factors (known to influence prognosis) that we examined had a significant association with elevated CRP. Therefore, it is unlikely that such confounding factors influenced our survival analysis. However, in future studies, it will be important to validate the refined prognostic cytokine profile in a larger group of patients undergoing resection of colorectal liver metastases. In addition, the independent contribution of this panel of biomarkers on outcomes will have to be evaluated with a multivariable survival analysis.

In addition to mGPS, a number of other indices of inflammation have been described, including neutrophil/ lymphocyte ratio (NLR), platelet/lymphocyte ratio (PLR), and prognostic nutritional index (PNI; a combination of albumin and lymphocyte count). It is presently unclear whether all of these indices represent the same type of inflammatory response (characterized by the same pattern of circulating cytokines). Moreover, it is not known what indices best reflect the inflammatory state associated with a poor prognosis. This may well vary between types of malignancies. In a group of pancreatic cancers with various stages of disease and undergoing diverse treatments, NLR was prognostic and other indices were not [44]. In hepatocellular carcinoma, mGPS performed better as a prognosticating tool than NLR and PNI [45]. This discrepancy may be related to differences in the nature of the inflammatory response associated with different malignancies. Therefore, further study is required to understand the molecular and disease-related causes of cancer-associated inflammation, including how that inflammatory state may differ between cancers.

An important question related to any studies focusing on prognostic factors for cancer is whether any factor that effectively categorizes individuals could be used for treatment stratification. For example, for primary colon cancer, adjuvant chemotherapy is recommended for stage III, and only high risk patients in stage II are typically considered for adjuvant therapy [46]. Similarly, many clinicians would advocate administration of neoadjuvant chemotherapy for high risk patients with colorectal liver metastases as a means to better select surgical candidates [47]. While higher risk patients have a greater likelihood of benefiting from systemic therapy, in our view, treatment stratification based on prognostic biomarkers is not the ultimate goal. Rather, efforts must be directed at understanding the underlying biology that leads to poor oncologic outcomes so that novel therapeutic targets can be identified.

While the cohort studied was small, our data confirm that mGPS is a reflection of the inflammatory state. Specifically, elevated CRP indicates a proinflammatory state with features of a Th1 response. We have also demonstrated that prognosis of resected CR liver metastases is affected by a number of additional inflammatory (and perhaps angiogenic) events. This study paves the way to more detailed (and targeted) interrogations on the role of inflammation in the biology of CRC. In particular, it will be important to understand the contributions of the molecular features of tumor that incite a proinflammatory response, as well as characteristics of the host which perhaps lead to susceptibility to inflammation. The host response associated with elevated CRP (including the metabolic response and effects on distant organs) must be better understood, to gain an understanding of why the proinflammatory state is so detrimental. These more advanced studies will not only add to our capability to prognosticate; understanding the molecular pathways that portend a poor prognosis may lead to development of novel therapeutic strategies.

In all, our studies adhere to the REMARK reporting guidelines for prognostic tumor markers [48].

\section{Conclusions}

In patients with colorectal liver metastases, elevated CRP is associated with increased circulating proinflammatory cytokines with features of a Th1 response. Elevated CRP is associated with a shorter disease-free and overall survival following resection. Other cytokines that influenced survival outcomes included IL-8, PDGF-AB/BB, eotaxin and IP-10.

\section{Additional file}

Additional file 1: Table S1. Mean and standard deviation of all inflammatory mediators analyzed.

\section{Competing interests}

The authors declare that they have no competing interests.

\section{Authors' contributions}

TDH participated in the experimental design and coordinated data analysis. $\mathrm{DL}$ and KK performed the statistical analysis. ED and FRS participated in the experimental design and aided in outcomes analysis. OFB conceived of the study, participated in the experimental design, and coordinated data analysis. TDH, KK and OFB drafted the manuscript. All authors read and approved the final copy of the manuscript.

\section{Acknowledgements}

The authors would like to thank Mark Fritzler (Eve Technologies Corporation) for performing the Luminex assay. This research was supported by funding from Calgary Laboratory Services and Tom Baker Cancer Centre.

\section{Author details}

${ }^{1}$ Department of Surgery, University of Calgary, Calgary, AB, Canada. ${ }^{2}$ Department of Mathematics and Statistics, University of Calgary, Calgary, $A B$, Canada. ${ }^{3}$ Department of Oncology, University of Calgary, 1331-29th St NW, Calgary T2N 4N2, AB, Canada.

Received: 4 July 2013 Accepted: 10 July 2014

Published: 28 July 2014 


\section{References}

1. Finlay IG, McArdle CS: Occult hepatic metastases in colorectal carcinoma. Br J Surg 1986, 73:732-735.

2. Bathe OF, Ernst S, Sutherland FR, Dixon E, Butts C, Bigam D, Holland D, Porter GA, Koppel J, Dowden S: A phase II experience with neoadjuvant irinotecan (CPT-11), 5-fluorouracil (5-FU) and leucovorin (LV) for colorectal liver metastases. BMC Cancer 2009, 9:1-10.

3. Pawlik TM, Scoggins CR, Zorzi D, Abdalla EK, Andres A, Eng C, Curley SA, Loyer EM, Muratore A, Mentha G, Capussotti L, Vauthey JN: Effect of surgical margin status on survival and site of recurrence after hepatic resection for colorectal metastases. Ann Surg 2005, 241:715-724.

4. Wei AC, Greig PD, Grant D, Taylor B, Langer B, Gallinger S: Survival after hepatic resection for colorectal metastases: a 10-year experience. Ann Surg Oncol 2006, 13:668-676.

5. Fong Y, Fortner J, Sun RL, Brennan MF, Blumgart LH: Clinical score for predicting recurrence after hepatic resection for metastatic colorectal cancer. Ann Surg 1999, 230:309-321.

6. Iwatsuki S, Dvorchik I, Madariaga JR, Marsh JW, Dodson F, Bonham AC, Geller DA, Gayowski TJ, Fung JJ, Starzl TE: Hepatic resection for metastatic colorectal adenocarcinoma: a proposal of a prognostic scoring system. J Am Coll Surg 1999, 189:291-299.

7. Konopke R, Kersting S, Distler M, Dietrich J, Gastmeier J, Heller A, Kulisch E, Saeger HD: Prognostic factors and evaluation of a clinical score for predicting survival after resection of colorectal liver metastases. Liver Int 2009, 29:89-102.

8. Lise M, Bacchetti S, Da Pian P, Nitti D, Pilati P: Patterns of recurrence after resection of colorectal liver metastases: prediction by models of outcome analysis. World J Surg 2001, 25:638-644.

9. Minagawa M, Yamamoto J, Kosuge T, Matsuyama Y, Miyagawa S, Makuuchi M: Simplified staging system for predicting the prognosis of patients with resectable liver metastasis. Arch Surg 2007, 142:269-276.

10. Nagashima I, Takada T, Matsuda K, Adachi M, Nagawa H, Muto T, Okinaga K: A new scoring system to classify patients with colorectal liver metastases: proposal of criteria to select candidates for hepatic resection. J Hepatobiliary Pancreat Surg 2004, 11:79-83.

11. Rees M, Tekkis PP, Welsh FKS, O'Rourke T, John TG: Evaluation of long-term survival after hepatic resection for metastatic colorectal cancer. Ann Surg 2008, 247:125-135.

12. Schindl M, Wigmore SJ, Currie EJ, Laengle F, Garden OJ: Prognostic scoring in colorectal cancer liver metastases. Arch Surg 2005, 140:183-189.

13. Ueno $H$, Mochizuki $H$, Hatsuse $K$, Hase $K$, Yamamoto T: Indicators for treatment strategies of colorectal liver metastases. Ann Surg 2000, 231:59-66.

14. McMillan DC, Crozier JEM, Canna K, Angerson WJ, McArdle CS: Evaluation of an inflammation-based prognostic score (GPS) in patients undergoing resection for colon and rectal cancer. Int J Colorectal Dis 2007, 22:881-886.

15. Forrest LM, McMillan DC, McArdle CS, Anderson WJ, Dunlop DJ: Evaluation of cumulative prognostic scores based on the systemic inflammatory response in patients with inoperable non-small-cell lung cancer. Br J Cancer 2003, 89:1028-1030

16. Ishizuka M, Nagata H, Takagi K, Horie T, Kubota K: Inflammation-based prognostic score is a novel predictor of postoperative outcome in patients with colorectal cancer. Ann Surg 2007, 246:1047-1051.

17. Roxburgh CSD, Salmond JM, Horgan PG, Oien KA, McMillan DC: Comparison of the prognostic value of inflammation-based pathologic and biochemical criteria in patients undergoing potentially curative resection for colorectal cancer. Ann Surg 2009, 249:788-793.

18. Kobayashi T, Teruya M, Kishiki T, Endo D, Takenaka Y, Miki K, Kobayashi K, Morita K: Elevated c-reactive protein and hypoalbuminemia measure before resection of colorectal liver metastases predict postoperative survival. Dig Surg 2010, 27:285-290

19. Wong VKH, Malik HZ, Hamady ZZR, Al-Mukhtar A, Gomez D, Prasad KR, Toogood GJ, Lodge JP: C-reactive protein as a predictor of prognosis following curative resection for colorectal liver metastases. $\mathrm{Br} J$ Cancer 2007, 96:222-225.

20. Ishizuka M, Kita J, Shimoda M, Rokkaku K, Kato M, Sawada T, et al: Systemic inflammatory response predicts postoperative outcome in patients with liver metastases from colorectal cancer. J Surg Onc 2009, 100:38-42.

21. Malik HZ, Prasad KR, Halazun KJ, Aldoori A, Al-Mukhtar A, Gomez D, Lodge JP, Toogood GJ: Preoperative prognostic score for predicting survival after hepatic resection for colorectal liver metastases. Ann Surg 2007, 246:806-814.
22. McMillan DC, Canna K, McArdle CS: Systemic inflammatory response predicts survival following curative resection of colorectal cancer. Br J Surg 2003, 90:215-219.

23. Shimada H, Nabeya Y, Okazumi S, Matsubara H, Shiratori T, Aoki T, Sugaya M, Miyazawa Y, Hayashi H, Miyazaki S, Ochiai T: Elevation of preoperative serum C-reactive protein level is related to poor prognosis in esophageal squamous cell carcinoma. J Surg Oncol 2003, 83:248-252.

24. Jamieson NB, Glen P, McMillan DC, McKay CJ, Foulis AK, Carter R, Imrie CW: Systemic inflammatory response predicts outcome in patients undergoing resection for ductal adenocarcinoma head of pancreas. Br J Cancer 2005, 92:21-23.

25. Al Murri AM, Wilson C, Lannigan A, Doughty JC, Angerson WJ, McArdle CS, McMillan DC: Evaluation of the relationship between the systemic inflammatory response and cancer-specific survival in patients with primary operable breast cancer. Br J Cancer 2007, 96:891-895.

26. Hilmy M, Bartlett JMS, Underwood MA, McMillan DC: The relationship between the systemic inflammatory response and survival in patients with transitional cell carcinoma of the urinary bladder. Br J Cancer 2005, 92:625-627.

27. Hefler LA, Concin N, Hofstetter G, Marth C, Mustea A, Sehouli J, Zeillinger R, Leipold H, Lass H, Grimm C, Tempfer CB, Reinthaller A: Serum C-reactive protein as independent prognostic variable in patients with ovarian cancer. Clin Cancer Res 2008, 14:710-714.

28. Karakiewicz PI, Hutterer GC, Trinh Q, Jeldres C, Perrotte P, Gallina A, Tostain $\mathrm{J}$, Patard J: C-reactive protein is an informative predictor of renal cell carcinoma-specific mortality. Cancer 2007, 110:1241-1247.

29. Friedman J, Hastie T, Tibshirani R: Regularization paths for generalized linear models via coordinate descent. J Stat Softw 2010, 33:1-22.

30. Simon N, Friedman J, Hastie T, Tibshirani R: Regularization paths for cox's proportional hazards model via coordinate descent. J Stat Softw 2011, 39:1-13.

31. Tusher VG, Tibshirani R, Chu G: Significance analysis of microarrays applied to the ionizing radiation response. Proc Natl Acad Sci 2001, 98:5116-5121

32. Chung YC, Chang YF: Significance of inflammatory cytokines in the progression of colorectal cancer. Hepatogastroenterology 2003, 50:1910-1913.

33. Ito $H$, Miki C: Profile of circulating levels of interleukin-1 receptor antagonist and interleuking-6 in colorectal cancer patients. Scand J Gastroenterol 1999, 11:1139-1143

34. Knupfer $H$, Preiss R: Serum interleukin-6 levels in colorectal cancer patients - a summary of published results. Int I Colorectal Dis 2010, 25:135-140.

35. Guthrie GJK, Roxburgh CSD, Richards CH, Horgan PG, McMillan DC: Circulating IL-6 concentrations link tumour necrosis and systemic and local inflammatory responses in patients undergoing resection for colorectal cancer. Br J Cancer 2013, 109:131-137.

36. Li A, Varney ML, Singh RK: Expression of interleukin 8 and its receptors in humon colon carcinoma cells with different metastatic potentials. Clin Cancer Res 2001, 7:3298-3304.

37. Waugh DJJ, Wilson C: The interleukin-8 pathway in cancer. Clin Cancer Res 2008, 14:6735-6741.

38. Peterson JE, Zurakowski D, Italiano JE, Michel LV, Connors S, Oenick M, D'Amato RJ, Klement GL, Folkman J: VEGF, PF4 and PDGF are elevated in platelets of colorectal cancer patients. Angiogenesis 2012, 15:265-273.

39. Cheadle EJ, Riyad K, Subar D, Rothwell DG, Ashton G, Batha H, Sherlock DJ, Hawkins RE, Gilham DE: Eotaxin-2 and colorectal cancer: a potential target for immune therapy. Clin Cancer Res 2007, 13:5719-5728.

40. Wagsater D, Lofgren S, Hugander A, Dienus O, Dimberg J: Analysis of single nucleotide polymorphism in the promoter protein expression of the chemokine Eotaxin-1 in colorectal cancer patients. World J Surg Oncol 2007, 5:84

41. Sgadari C, Angiolillo A, Cherney B, Pike SE, Farber JM, Koniaris LG, Vanguri $P$, Burd PR, Sheikh N, Gupta G, Teruya-Feldstein J, Tosato G: Interferon-inducible protein-10 identified as a mediator of tumor necrosis in vivo. Proc Natl Acad Sci 1996, 93:13791-13796.

42. Reckamp K, Figlin R, Moldawer N, Pantuck AJ, Belldegrun AS, Burdick MD, Strieter RM: Expression of CXCR3 on mononuclear cells and CXCR3 ligands in patients with metastatic renal cell carcinoma in response to systemic IL-2 therapy. J Immunother 2007, 30:417-424.

43. Jiang $Z, X u Y, C a i \mathrm{~S}:$ CXCL10 expression and prognostic significance in stage II and III colorectal cancer. Mol Biol Rep 2010, 37:3029-3036. 
44. Wang D, Luo H, Qiu M, Wang Z, Zhang D, Wang F, Li YH, Xu RH: Comparison of the prognostic values of various inflammation based factors in patients with pancreatic cancer. Med Oncol 2012, 29:3092-3100.

45. Kinoshita A, Onoda H, Imai N, Iwaku A, Oishi M, Fushiya N, Koike K, Nishine $\mathrm{H}$, Tajiri H: Comparison of the prognostic value of inflammation-based prognostic scores in patients with hepatocellular carcinoma. Br J Cancer 2012, 107:988-993.

46. Benson $A B$, Schrag $D$, Somerfield MR, Cohen AM, Figueredo AT, Flynn PJ, Krzyzanowska MK, Maroun J, McAllister P, Van Cutsem E, Brouwers M, Charette M, Haller DG: American Society of Clinical Oncology recommendations on adjuvant chemotherapy for stage II colon cancer. J Clin Oncol 2004, 22:3408-3419.

47. Adam R, Pascal G, Castaing D, Azoulay D, Delvart V, Paule B, Levi F, Bismuth $\mathrm{H}$ : Tumor progression while on chemotherapy: a contraindication to liver resection for multiple colorectal liver metastases? Ann Surg 2004, 240:1052-1061.

48. Altman DG, MCShane LM, Sauerbrei W, Taube SE: Reporting recommendations for tumor marker prognostic studies (REMARK): explanation and elaboration. BMC Med 2012, 10:51.

doi:10.1186/1471-2407-14-542

Cite this article as: Hamilton et al:: Identification of prognostic inflammatory factors in colorectal liver metastases. BMC Cancer 2014 14:542.

\section{Submit your next manuscript to BioMed Central and take full advantage of:}

- Convenient online submission

- Thorough peer review

- No space constraints or color figure charges

- Immediate publication on acceptance

- Inclusion in PubMed, CAS, Scopus and Google Scholar

- Research which is freely available for redistribution 